\title{
Crawling towards Building Smart Search Engine
}

\author{
Aparna Hambarde ${ }^{1}$, Shwetal Yadav², Aishwarya Dhekane ${ }^{3}$, Dipali Yadav ${ }^{4}$, Mayuri Pawar $^{5}$ \\ Professor, Computer Dept, KG College, Pune, India ${ }^{1}$ \\ Student, Computer Dept, KG College, Pune, India ${ }^{2,3,4,5}$
}

\begin{abstract}
The World wide web is the largest collection of statistics in these days and it maintains increasing each day. An web crawler is a program from the huge downloading of web pages from global huge web and this manner is known as web crawling. To gather the web pages from www a search engine makes use of web crawler and the web crawler collects this by using web crawling. Because of barriers of community bandwidth, time-eating and hardware's a web crawler can't down load all the pages, it's miles important to pick out the maximum important ones as early as possible throughout the crawling manner and avoid downloading and touring many beside the point pages. We used web page rank algorithm for powerful searching over internet. This paper opinions help the researches on web crawling techniques used for looking.
\end{abstract}

Keywords: Crawler, Database, Search Engine, World Wide Web.

\section{INTRODUCTION}

With the explosive boom of facts sources to be had on the world wide web, it has turn out to be vital to use computerized tools for locating the desired information assets, and for monitoring and reading their usage styles. For example if a user wished to find facts on the web then both had to know the proper deal with of the documents he sought or had to navigate patiently from hyperlink to hyperlink in hopes of locating his destination. These elements give upward thrust to the need of creating serverside and client-side sensible structures that could correctly mine for knowledge. Search engines like google will serve our cause. Search engines like google and yahoo include essential additives - web crawlers, a good way to discover, down load, and parse content material in the www and statistics miners, so one can extract keywords from pages, rank record importance and answer user queries. A web crawler (also called an internet spider or internet robotic) is a program or computerized script which browses the world wide web in a methodical, automated manner. This manner is called webcrawling or spidering. Many legitimate sites, in particular search engine, use spidering as a method of presenting up-to-date records. A web crawler is a internet bot which systematically browses the arena huge web, commonly for the purpose of web indexing (internet spidering). An web crawler starts with a list of urls to visit, called the seeds. Web crawlers (additionally known as as spiders, robots, walkers and wanderers) are applications which traverse through the internet attempting to find the relevant facts using algorithms that slender down the quest via locating out the most nearer and relevant information.

\section{LITERATURE SURVEY}

[1] M. Gray, "Internet Growth and Statistics: Credits and Background," available http://www.mit.edu/people/mkgray/net/backgrou nd.html.
Matthew Gray implemented the World Wide Web Wanderer $[2,20]$. It was written in Perl and was able to indexed pages from around 6000 sites.

[2] M. Mauldin, "Lycos: Design Choices in an Internet Search Service," IEEE Expert, vol. 12, pp. 8-11, 1997.

Another crawler named Lycos [3, 20] was developed that ran on a single machine and used Perl's associative arrays to maintain the set of URLs to crawl. It was capable to index tens of millions of pages; however, the design of this crawler remains undocumented.

[3] M. Burner, "Crawling towards Eternity: Building an Archive of the World Wide Web," Web Techniques Magazine, vol. 2, pp. 37-40, 1997.

Mike Burner's developed Internet Archive crawler [4, 20] that used multiple machines to crawl the web. Each crawler process was assigned up to 64 sites to crawl and no sites are assigned to more than one crawler. Each crawler process (single-threaded) read a list of seed URLs for its assigned sites from disk into per-site queues, and then used asynchronous I/O instructions to fetch pages from these queues in parallel. Once a page gets downloaded, the crawler extracted all the links contained in it.

[4] S. Brin, L. Page. "The Anatomy of a Large-scale Hyper textual Web Search Engine", International World Wide Web Conference, pp. 107-117, 1998.

The original Google crawler [5, 20] (developed at Stanford) consisted of five functional components running in different processes. A URL server process read URLs from a file and forwarded all to the multiple crawler processes. Each crawler process (single-threaded) that was running on a different machine used asynchronous $\mathrm{I} / \mathrm{O}$ instructions to fetch data from up to 300 web servers in parallel. Then all the crawlers transmitted downloaded 
pages to a single Store Server process that compressed the pages and stored them to the disk.

Identify the functions as $\mathrm{F}^{6}$

$\mathrm{S}=\{\ldots$

$\mathrm{F}=\{\mathrm{F} 1(), \mathrm{F} 2(), \mathrm{F} 3(), \mathrm{F} 4(), \mathrm{F} 5(), \mathrm{F} 6()\}$

[5] A. Heydon, M. Najork, "Mercator: A Scalable, Extensible Web Crawler, "World Wide Web, vol. 2, pp. 219-229, 1999.

Mercator was highly scalable and easily extensible crawler. It was written in Java. The first version [6] was non-distributed;

\section{PROPOSED SYSTEM}

Web crawlers recursively traverse and down load net pages (the usage of GET and POST instructions) for engines like google to create and keep the net indices. The need for maintaining the as up to-date pages cause a crawler to revisit the web sites again and again. A crawler that's from time to time mentioned spider, bot or agent is software whose reason, it has done web crawling. This could be used for accessing the net pages from the internet server as in step with person bypass queries commonly for seek engine. A web crawler also used sitemap protocol for crawling net pages. In the crawling technique, normally starts with a fixed of uniform useful resource locator (urls) known as the seed url. In standard, it starts with a list of urls to visit, called seed urls. Because the crawler traverses these urls, it identifies all links inside the web page and adds them to the list of urls to be visited, referred to as the crawl frontier. Urls from the crawl frontier are visited separately and looking of the enter sample is accomplished every time text content is extracted from the web page source of the internet web page.

\section{ARCHITECTURE DIAGRAM}

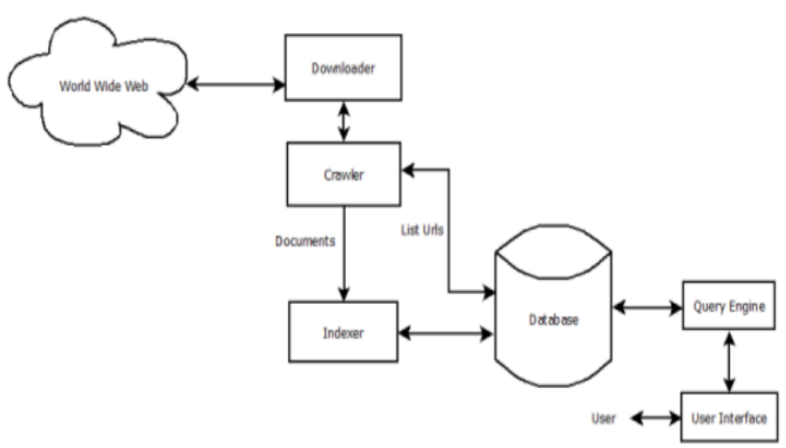

V. MATHEMATICAL MODEL

Let_S' be the $\mid$ system in the final set

$\mathrm{S}=\{\ldots \ldots \ldots \ldots\}$

Identify the inputs as I

$\mathrm{S}=\{\mathrm{K}\}$

$\mathrm{K}=\{\mathrm{k} 1, \mathrm{k} 2, \mathrm{k} 3, \mathrm{k} 4 \ldots \mid \mathrm{K}$ is set of keywords enter by users $\}$

Identify the outputs as $\mathrm{O}$

$\mathrm{S}=\{\mathrm{URL}\}$

$\mathrm{URL}=\{\mathrm{URL} 1, \mathrm{URL} 2, \mathrm{URL} 3, \ldots \ldots \ldots . . \mathrm{URL}$ is selected URL

by system $\}$
$\mathrm{F} 1(\mathrm{~V})=$ User will enter new keyword

F2 $(\mathrm{V})=$ Keyword will be matched with keyword already present in data base

$\mathrm{F} 3(\mathrm{~V})=$ Sorting process is done

F4 $(\mathrm{V})=$ Display top $10 \mathrm{URL}$

F5 $(\mathrm{V})=$ Display doc file

F6 (V) =Colour nouns, verb, pronoun with different colour.

\section{CONCLUSION}

Size of the net is developing and from consumer point of view, consumer expects the crawler to retrieve the consequences as soon as feasible. Due to the dynamic way of the internet, the association of pertinent pages for any given question is moreover profoundly effective, prompting a scalability problem is the supposition of a precise and whole static photograph of the net separates with its charge of progress. As search fail to satisfy the person's requirement for whole and currently up to date data, it seems to be profoundly appealing to utilize dispensed net crawlers.

\section{REFERENCES}

[1] S. Pavalam, M. Jawahar, F. Akorli, S. Raja,Web Crawler in Mobile Systems, IJMLC, vol. 2, pp. 531-534.

[2] M. Gray, Internet Growth and Statistics: Credits and Background, available http://www.mit.edu/people/mkgray/net/background.html

[3] M. Mauldin, Lycos: Design Choices in an Internet Search Service, IEEE Expert, vol. 12, pp. 8-11, 1997.

[4] M. Burner, Crawling towards Eternity: Building an Archive of the World Wide Web, Web Techniques Magazine, vol. 2, pp. 37-40, 1997.

[5] S. Brin, L. Page. The Anatomy of a Large-scale Hyper textual Web Search Engine, International World Wide Web Conference, pp. 107-117,1998.

[6] Heydon, M. Najork, Mercator: A Scalable, Extensible Web Crawler, World Wide Web, vol.2, pp. 219-229, 1999.

[7] M. Najork, A. Heydon, High-performanceWeb Crawling, Technicalreport, Compaq SRC Research Report 173, 2001.

[8] C. Aggarwal, Mining text streams, in Mining Text Data, Springer US,2012, pp. 297321.

[9] J.-Y. Nie et al., Translingual mining from text data, in Mining Text Data, Springer US, 2012, pp. 323359.

[10] Y. Sun et al., Probabilistic models for text mining, in Mining Text Data, Springer US, 2012, pp. 259295.

[11] W. Pan et al, Transfer learning for text mining, in Mining Text Data, Springer US, 2012, pp. 223257.

[12] C. Aggarwal and C. Zhai, A survey of text clustering algorithms, in Mining Text Data, Springer US, 2012, pp. 77128.

[13] Nenkova and K.McKeown, A survey of text summarization techniques, $\mathrm{n}$ in Mining Text Data, Springer US, 2012, pp. 4376. 